\title{
Microbiological Profile and Antibiogram in Cases of Chronic Suppurative Otitis Media at a Tertiary Care Hospital, Jaipur, India
}

\author{
Jitendra* and Shiv Kumar \\ Department of Microbiology, NIMS Medical College, Jaipur, Rajasthan, India \\ *Corresponding author
}

\begin{tabular}{|l|}
\hline K e y w o r d s \\
Chronic Suppurative \\
Otitis Media (CSOM), \\
Children, Ear \\
Discharge, \\
Otomycosis \\
Pseudomonas \\
aeruginosa, \\
Staphylococcus \\
aureus, Aspergillus \\
niger
\end{tabular}

A B S T R A C T

Otitis media is an inflammation of the middle ear cleft without reference to etiology or pathogenesis. Otitis media is more common in children, as their Eustachian Tube is shorter, narrower and more horizontal than the adult ear. Therefore, the study of microorganisms commonly associated with CSOM and their antibiotic sensitivity pattern is vital for the clinician to plan a general outline of treatment for the patient with a chronically discharging ear. Hence the aim of our study is to find out the fungal and bacterial aetiology and their antibiotic susceptibility pattern in clinically diagnosed CSOM cases in tertiary care hospital at Jaipur. The present study was carried on total of 53 clinically diagnosed CSOM cases attending ENT Department. After proper sample collection by sterile cotton swabs, they were immediately sent to the microbiology laboratory for aerobic bacterial culture, isolation and identification Conventional bacteriological and Mycological methods were used for identification of CSOM and susceptibility testing was performed as per CLSI guidelines 2017 . This study comprises of 45 isolates of CSOM from total of 53 clinical specimens, collected from in- patients attended to ENT department during a period of 6 months. The most common organism causing CSOM among aerobic bacteria were Pseudomonas species i.e. $16(29 \%)$ then Staphylococcus aureus 11(20\%). In fungi most common organism isolated was Aspergillus niger then Candida albicans. Meropenem was most effective 100\% against gram negative bacilli. In Pseudomonas Ticarcillin/Clavulanic Acid, Ceftazidime, Colistin and Polymyxin-B were $100 \%$ sensitive. Linezolid, Vancomycin and Rifampicin were $100 \%$ effective against gram positive cocci. The periodic evaluation of microbiological pattern and their antibiotic sensitivity pattern in local area becomes important and helpful in prescribing empirical antibiotics for successful treatment of CSOM and thus minimizing its complications and emergence of resistance strains.

\section{Introduction}

Otitis media is an inflammation of the middle ear cleft without reference to etiology or pathogenesis (Agrawal et al., 2013). Otitis media is more common in children, as their
Eustachian tube is shorter, narrower and more horizontal than the adult ear; and children, are at this age more prone to frequent upper respiratory tract infections which ascends to middle ear and may subsequently progress to CSOM. The difference in anatomy of the 
paediatric ET favours creation of stasis of nasopharyngeal secretions making their movement difficult favouring the growth of microorganism than in adults (Bluestone et al., 1988).

The acute form is acute suppurative otitis media usually associated with the infection in the upper respiratory tract whereas persistent form is known as chronic suppurative otitis media (CSOM) (Berman et al., 1995) (Bluestone et al., 1988).

Acute Suppurative otitis media is an acute inflammation of middle ear by pyogenic organism and is more common especially in infants and children of lower socioeconomic group (Dhingra et al., 2004).

Chronic Suppurative Otitis Media is characterized by drainage from the middle ear for at least two weeks and is associated with a tympanic membrane perforation that is usually painless (Dhingra et al., 2004).

The disease is mainly classified into two types (1) Mucosal or Tubotympanic type affecting the middle ear mucosa also called as 'safe disease', as it is characterized by a central perforation of the para tensa. (2) Squamosal or Atticoantral type which is an active squamous disease in which squamous epithelium present in middle ear cleft erodes the bone.

Bone erosion, with potentially dangerous results, was an inherent pathological features. Another synonym has been erosive middle ear disease and also called as 'Unsafe disease'.

The incidence of CSOM is higher in developing countries, especially among the low socioeconomic status of the society, use of poor nutrition, improper hygiene and lack of health education (Kumar et al., 2011). The disease is highly prevalent in tropical regions including south Asia (Parveen et al., 2012).
CSOM has direct impact on the hearing of patient causing conductive and sensorineural hearing loss and also on child development. It is also found to be the single major cause for conductive deafness, and is responsible for $1.5 \%$ speech disorders. Hearing loss associated with CSOM hampers educational skills in children that are well recognized by otologists, paediatricians and educators (Dhingra et al., 2004).

The most common organism isolated now a days are Pseudomonas aeruginosa, Staphylococcus aureus, Streptococcus pyogenes, Proteus species, Klebsiella species among aerobes and among anaerobes Bacteriodes, Peptostreptococcus, Proprionibacterium (Barobby et al., 1987).

Most common fungi are Aspergillus species and Candida species (Park et al., 2008).

The diagnosis of COM is based on history, examination findings on otoscopy, supplemented by culture of the ear discharge and radiology of the temporal bone. Gram stains and cultures will assist in guiding therapy and are usually reserved for cases that fail standard topical therapy.

Computed tomography (CT) scan should be performed if extra cranial complications are suspected. CT scan has a role in diagnosis of COM when the TM cannot be visualized, for example by narrowing or stenosis of the EAC. It may reveal bone erosion from a cholesteatoma or point to area of potential fistula formation. MRI particularly delineates intracranial pathology that complicates CSOM (Roland et al., 1999) (Jung et al., 2006).

Antibiotics are used to treat the infections but most of the organisms are acquiring resistance. In developing countries this problem is increasing rampantly due to misuse of antibiotics and inadequate antibiotic 
treatment. Hence the aim of our study is to find out the fungal and bacterial aetiology and their antibiotic susceptibility pattern so as to know whether an empirical treatment based on the result of the study will effectively shorten the period of infection associated with discharge in patients of CSOM and thereby improve the surgical outcome.

\section{Materials and Methods}

This Descriptive cross sectional study was carried out in the Department of Microbiology of National Institute of Medical Sciences (NIMS) University, Jaipur, Rajasthan, India for a period of six months (February 2017 to July 2017). A total of 53 clinically diagnosed cases of CSOM attending ENT department of NIMS medical college was taken. Data regarding patient age, sex, duration of illness, previous medications and ears involved was obtained from the patient. The organisms isolated and identified provisionally as CSOM were identified further by using a standard protocol for identification (Koneman et al., 2006).

\section{Materials and Methods}

\section{Inclusion criteria}

Adult and paediatric patients of both sexes and all age group who attend with the ear discharge of more than 2 weeks duration.

\section{Exclusion criteria}

Patient on systemic or topical antibiotics, patient with acute Suppurative otitis media i.e. duration of less than 2 weeks, Patients with cleft palate, Patients /parent /care giver who does not give his or her consent.

Isolation of various isolates was confirmed by several of test like colony characters, morphology (Gram's stain), pigment production, motility (by hanging drop) and other biochemical tests like Catalase, Oxidase test, Indole, Methyl red, Voges Proskauer, Citrate utilization test, Urease test and Triple sugar iron Test, Oxidation/fermentation for glucose, lactose, xylose, mannitol and maltose (Hugh and Leifson's media), Lysine and Ornithine decarboxylase and Arginine dihydrolase activity, ONPG test, Esculin test etc. were done for isolation of the gram negative and positive bacteria.

The following studies are necessary for isolation of fungi

Microscopic examination of sample by Gram staining and $\mathrm{KOH}$ Mount (Chandra, 2008)

\section{Fungal culture}

Sample was inoculated on SDA at $25^{\circ} \mathrm{C}$ and $37^{\circ} \mathrm{C}$ and was observed daily for fungal growth up to 4 weeks (Chandra, 2008). The identification of species of fungal growth was done by various methods like Morphology of colony, Texture, Surface pigmentation, Microscopic examination like LPCB mount and slide culture (on chrome agar) were done to identify the fungi. Gram staining was done for identification of yeast and yeast like fungi, Chlamydospore formation and germ tube tests were done to identify Candida albicans, growth on corn meal agar.

\section{Antimicrobial susceptibility}

Antibiotic susceptibility testing was done by Modified Kirby Bauer's disc diffusion method as per CLSI guidelines 2017 using commercially available discs.

\section{Results and Discussion}

A total of 53 clinically diagnosed CSOM cases attending ENT department of NIMS medical college, Jaipur during the study 
period were taken. In the present study, most of the CSOM cases i.e. 47 (89\%) had unilateral ear involvement and only $6(11 \%)$ had bilateral ear involvement. In patients with unilateral ear involvement most predominantly right ear i.e. 33(62\%) patients were infected whereas left ear was involved in only 14 (27\%) CSOM cases (Table 1 and 2).

In the current study, CSOM infection was found comparatively more in females patients $34(64 \%)$ than in male patients $19(36 \%)$. The male to female ratio was $1: 1.8$. Age group of $11-20$ years $(32 \%)$ and $21-30$ years $(21 \%)$ were predominantly affected (Table 3 ).

In the present study out of 53 ear swabs processed microbial growth was in $45(85 \%)$ while $8(15 \%)$ samples showed no growth (Table 4).

Out of 53 patients, 45 showed growth of bacteria and fungi. Pure growth (Monomicrobial) was obtained from 36 (68\%) patients whereas 9 (17\%) patients showed mixed growth (Polymicrobial) (Table 5).

Out of these 36 patients majority 32 (89\%) showed pure bacterial growth only and 4 (11\%) showed pure fungal growth (Table 6).

However, in remaining $9(17 \%)$ patients mixed growth was obtained in which $2(4 \%)$ patients showed mixed bacterial growth and 7
(13\%) patients showed mixed bacterial and fungal growth. In the present study, most common organism isolated was Pseudomonas species (29\%) then Staphylococcus aureus $20 \%$. This was followed by CONS (12.50\%), Aspergillus niger (9\%), Escherichia coli (5\%), Candida albicans (5\%), Proteus mirabilis (3.57\%), Proteus vulgaris (3.57\%), Klebsiella spp. (3.57\%), Citrobacter (1.79\%), Non fermentative (1.79\%), Candida tropicalis (1.79\%), Rhizopus (1.79\%) and Alterneria (1.79\%) (Table 7).

In GPC most common organism isolated was Staphylococcus aureus 11(20\%) followed by CONS 7(12.50\%).

In GNB Most common organism isolated was Escherichia coli (5\%) followed by Proteus mirabilis (3.57\%), Proteus vulgaris $(3.57 \%)$, Klebsiella spp. (3.57\%), Citrobacter (1.79\%).

In Pseudomonas species most common organism isolated was Pseudomonas aeruginosa 5(8.93\%) followed by Pseudomonas species 11 (20\%) and Nonfermentative $1(1.79 \%)$.

In fungal most common organism isolated was Aspergillus niger 5(8.93\%) them Candida albicans 3 (5.36\%) followed by Candida tropicalis (1.79\%), Rhizopus (1.79\%) and Alterneria (1.79\%) (Fig. 1 and 2).

Table.1 Various comparative studies on side of ear involved in CSOM cases

\begin{tabular}{|l|l|l|l|l|l|}
\hline Side involved & $\begin{array}{l}\text { Present study } \\
(\mathbf{2 0 1 7})\end{array}$ & $\begin{array}{l}\text { Ramesh Agarwal } \\
\text { et al., (2017) }\end{array}$ & $\begin{array}{l}\text { Jeyakumari et } \\
\text { al., (2015) }\end{array}$ & $\begin{array}{l}\text { Ruby Naz et al., } \\
(\mathbf{2 0 1 5})\end{array}$ & $\begin{array}{l}\text { Rakesh kumar } \\
\text { et al., (2013) }\end{array}$ \\
\hline Unilateral & $89 \%$ & $89.34 \%$ & $93 \%$ & $73 \%$ & $79 \%$ \\
\hline Bilateral & $11 \%$ & 11 & $7 \%$ & $27 \%$ & $21 \%$ \\
\hline
\end{tabular}

Table. 2 Various comparative studies on predominant side of infection in patients with unilateral ear involvement

\begin{tabular}{|l|l|l|l|}
\hline Unilateral ear involvement & Present study (2017) & B.L. Choudhary et al., (2014) & R Shyamala et al., (2012) \\
\hline Right ear & $62 \%$ & $60 \%$ & $62 \%$ \\
\hline Left ear & $27 \%$ & $34 \%$ & $33 \%$
\end{tabular}


Table.3 Various comparative studies on sex wise distributions of patients with CSOM infection

\begin{tabular}{|l|l|l|l|l|l|}
\hline Gender & $\begin{array}{l}\text { Present study } \\
(2017)\end{array}$ & $\begin{array}{l}\text { Ramesh Agarwal } \\
\text { et al., (2017) }\end{array}$ & $\begin{array}{l}\text { Ruby Naz et } \\
\text { al., (2015) }\end{array}$ & $\begin{array}{l}\text { Sushmita et al., } \\
(\mathbf{2 0 1 4})\end{array}$ & $\begin{array}{l}\text { Prakash M et } \\
\text { al., (2013) }\end{array}$ \\
\hline Male & $36 \%$ & $58 \%$ & $47 \%$ & $58 \%$ & $45 \%$ \\
\hline Female & $64 \%$ & $42 \%$ & $53 \%$ & $42 \%$ & $55 \%$ \\
\hline
\end{tabular}

Table.4 Various comparative studies on rate of isolation of microbial growth from CSOM cases

\begin{tabular}{|l|l|l|l|l|l|}
\hline Isolation rate & $\begin{array}{l}\text { Present } \\
\text { study }\end{array}$ & $\begin{array}{l}\text { Yadav Saurabh et } \\
\text { al., (2016) }\end{array}$ & $\begin{array}{l}\text { Dhirendra kumar } \\
\text { et al., (2016) }\end{array}$ & $\begin{array}{l}\text { Nawaz umar } \\
\text { et al., (2015) }\end{array}$ & $\begin{array}{l}\text { Sunil kumar et } \\
\text { al., (2015) }\end{array}$ \\
\hline Microbial growth & $85 \%$ & $93 \%$ & $86 \%$ & $88.6 \%$ & $82 \%$ \\
\hline No growth & $15 \%$ & $7 \%$ & $14 \%$ & $14.4 \%$ & $18 \%$ \\
\hline
\end{tabular}

Table.5 Various comparative studies on type of infection in CSOM cases

\begin{tabular}{|l|l|l|l|l|l|}
\hline Infection & $\begin{array}{l}\text { Present } \\
\text { study }\end{array}$ & $\begin{array}{l}\text { Yadav saurabh } \\
\text { et al., (2016) }\end{array}$ & $\begin{array}{l}\text { Raakhee T et } \\
\text { al., (2014) }\end{array}$ & $\begin{array}{l}\text { Kusuma Bai S } \\
\text { et al., (2013) }\end{array}$ & $\begin{array}{l}\text { Prayaga N et } \\
\text { al., (2013) }\end{array}$ \\
\hline Monomicrobial & $68 \%$ & $85 \%$ & $70 \%$ & $66 \%$ & $39 \%$ \\
\hline Polymicrobial & $17 \%$ & $8 \%$ & $15 \%$ & $31 \%$ & $11 \%$ \\
\hline
\end{tabular}

Table.6 Various comparative studies on microorganism isolated from CSOM cases

\begin{tabular}{|l|c|c|c|c|}
\hline Microorganism & Present study & $\begin{array}{c}\text { Ramesh Agrawal } \\
\text { et al., (2017) }\end{array}$ & $\begin{array}{c}\text { Asish J et al., } \\
{[88](2013)}\end{array}$ & $\begin{array}{c}\text { Shrestha } \text { et } \\
\text { al.,[126] (2011) }\end{array}$ \\
\hline GNB & $48 \%$ & $46 \%$ & $64 \%$ & $51 \%$ \\
\hline GPC & $32 \%$ & $47 \%$ & $30 \%$ & $38 \%$ \\
\hline Fungus & $20 \%$ & $7 \%$ & $6 \%$ & $11 \%$ \\
\hline
\end{tabular}

Table.7 Comparative studies on various bacterial and fungal species isolated from CSOM cases

\begin{tabular}{|l|}
\hline Organism \\
\hline Pseudomonas spp. \\
\hline $\begin{array}{l}\text { Staphylococcus } \\
\text { aureus }\end{array}$ \\
\hline $\begin{array}{l}\text { Coagulase Negative } \\
\text { Staph. aureus }\end{array}$ \\
\hline E.coli \\
\hline Citrobacter \\
\hline Proteus Mirabilis \\
\hline Proteus Vulgaris \\
\hline Klebsiella spp. \\
\hline Non fermenters \\
\hline Candida albicans \\
\hline Aspergillus spp. \\
\hline Candida tropicalis \\
\hline Rhizopus spp. \\
\hline Alterneria \\
\hline
\end{tabular}

\begin{tabular}{|c|c|}
\multicolumn{1}{l|}{$\begin{array}{l}\text { Present } \\
\text { study }\end{array}$} & $\begin{array}{l}\text { Geeta S.H. et } \\
\text { al., (2014) }\end{array}$ \\
\hline 28.57 & 17.92 \\
\hline 19.64 & 19.91 \\
\hline 12.50 & 2.83 \\
\hline 5.36 & 1.26 \\
\hline 1.79 & 6.28 \\
\hline 3.57 & 9.12 \\
\hline 3.57 & 1.57 \\
\hline 3.57 & 3.14 \\
\hline 1.79 & 2.20 \\
\hline 5.36 & 1.57 \\
\hline 8.93 & 15.14 \\
\hline 1.79 & 1.88 \\
\hline 1.79 & 0.63 \\
\hline 1.79 & - \\
\hline
\end{tabular}

\begin{tabular}{|c|c|c|c|}
\hline $\begin{array}{l}\text { Ramesh } \\
\text { Agrawal et al., } \\
(\mathbf{2 0 1 7})\end{array}$ & $\begin{array}{l}\text { Dhirendra } \\
\text { kumar et al., } \\
(2016)\end{array}$ & $\begin{array}{l}\text { G } \\
\text { Lakshmi et al., } \\
(2014)\end{array}$ & $\begin{array}{l}\text { Ashish J et } \\
\text { al., (2013) }\end{array}$ \\
\hline $41.1 \%$ & $26 \%$ & $41 \%$ & $33 \%$ \\
\hline $38.7 \%$ & $38 \%$ & $28.2 \%$ & $25.8 \%$ \\
\hline $8.5 \%$ & - & $2.5 \%$ & - \\
\hline $2.3 \%$ & $6 \%$ & $5.1 \%$ & - \\
\hline $1.5 \%$ & $1.5 \%$ & $2.5 \%$ & - \\
\hline $1.5 \%$ & $7 \%$ & - & $20.6 \%$ \\
\hline $3.1 \%$ & $7 \%$ & $7.6 \%$ & - \\
\hline- & $6 \%$ & $3.8 \%$ & $4.1 \%$ \\
\hline- & - & - & - \\
\hline $2.3 \%$ & - & $1.3 \%$ & $1 \%$ \\
\hline $5.4 \%$ & - & $3.8 \%$ & $5.2 \%$ \\
\hline- & - & - & - \\
\hline- & - & - & - \\
\hline- & - & - & - \\
\hline
\end{tabular}


Table.8 Comparative studies on antibiotic sensitivity pattern of Staphylococcus aureus isolated from CSOM cases

\begin{tabular}{|l|l|l|l|l|l|}
\hline Antibiotics & $\begin{array}{l}\text { Present } \\
\text { Study }\end{array}$ & $\begin{array}{l}\text { Ramesh } \\
\text { Agrawal } \text { et } \\
\text { al., (2017) }\end{array}$ & $\begin{array}{l}\text { Dhirendra } \\
\text { kumar } \\
\text { al., (2016) }\end{array}$ & $\begin{array}{l}\text { Jeyakumari } \\
\text { et al., (2015) }\end{array}$ & $\begin{array}{l}\text { Rakesh } \\
\text { kumar } \text { et al., } \\
(\mathbf{2 0 1 3})\end{array}$ \\
\hline Erythromycin & 36 & 56 & 66.7 & 67 & 45 \\
\hline Clindamycin & 73 & 94 & 85.2 & 93 & 70 \\
\hline Cotrimoxazole & 55 & - & 83.3 & 33 & - \\
\hline Penicillin & 9 & 26 & - & 7 & - \\
\hline Tetracycline & 91 & 94 & - & - & - \\
\hline Linezolid & 100 & 98 & 100 & 100 & 100 \\
\hline Vancomycin & 100 & 90 & 100 & 100 & 100 \\
\hline Rifampicin & 100 & - & - & - & - \\
\hline Ciprofloxacin & 55 & 72 & - & 73 & 30 \\
\hline Gentamycin & 91 & - & - & - & -
\end{tabular}

Table.9 Comparative studies on MRSA prevalence in S. aureus isolates

\begin{tabular}{|l|l|l|l|l|l|l|} 
& $\begin{array}{l}\text { Present } \\
\text { study }\end{array}$ & $\begin{array}{l}\text { Ramesh } \\
\text { Agrawal } \\
\text { et al., } \\
(\mathbf{2 0 1 7})\end{array}$ & $\begin{array}{l}\text { Upasana } \\
\text { Bhumbla et., (2016) }\end{array}$ & $\begin{array}{l}\text { Dhirendra } \\
\text { kumar et } \\
\text { al., (2016) }\end{array}$ & $\begin{array}{l}\text { Bansal } \\
\text { sulabh } \\
\text { et al., } \\
(\mathbf{2 0 1 3})\end{array}$ & $\begin{array}{l}\text { Chakraborty } \\
\text { et al., (2013) }\end{array}$ \\
\hline MRSA & $27.27 \%$ & $88 \%$ & $35.7 \%$ & $7 \%$ & $27.7 \%$ & $34.4 \%$ \\
\hline
\end{tabular}

Table.10 Comparative studies on antibiotic sensitivity pattern of Pseudomonas species isolated from CSOM cases

\begin{tabular}{|l|l|l|l|l|l|}
\hline Antibiotics & $\begin{array}{l}\text { Present } \\
\text { study }\end{array}$ & $\begin{array}{l}\text { Ramesh } \\
\text { Agrawal et } \\
\text { al., (2017) }\end{array}$ & $\begin{array}{l}\text { Dhirendra } \\
\text { kumar et } \\
\text { al., (2016) }\end{array}$ & $\begin{array}{l}\text { Upasana } \\
\text { Bhumbla } \\
\text { al., (2016) }\end{array}$ & $\begin{array}{l}\text { Ruby Naz et } \\
\text { al., (2015) }\end{array}$ \\
\hline Cefepime & 65 & - & 86.8 & & \\
\hline Amikacin & 65 & 83 & 71.1 & 66.7 & 75 \\
\hline Ticarcillin/Clavulanic Acid & 94 & - & - & & - \\
\hline Aztreonam & 88 & 53 & - & & - \\
\hline Ciprofloxacin & 65 & 73 & 76.3 & 30.4 & 75 \\
\hline Ceftazidime & 100 & 24 & 71.1 & 61.1 & 53 \\
\hline Meropenem & 82 & 94 & 84.2 & 86.1 & 87 \\
\hline Gentamycin & 88 & - & 73.7 & 27.3 & 81 \\
\hline Piperacillin & 88 & 85 & - & & - \\
\hline Piperacillin tazobactum & 76 & - & 97.4 & 63.6 & 81 \\
\hline Colistin & 88 & - & - & & 93 \\
\hline Polymyxin- B & 82 & - & 94.7 & & 87
\end{tabular}


Table.11 Comparative studies on Antibiotic sensitivity pattern of E. coli isolated from CSOM cases

\begin{tabular}{|l|}
\hline Antibiotics \\
\hline Ceftaridime \\
\hline Ceftaridime/Clavunic acid \\
\hline Meropenam \\
\hline Gentamicin \\
\hline Cefepime \\
\hline Ampicillin \\
\hline Amikacin \\
\hline Piperacillin \\
\hline Piperacillin/Tazobactum \\
\hline Cefazolin
\end{tabular}

\begin{tabular}{|l|l|l|l|}
\hline Present study & $\begin{array}{l}\text { Ramesh Agrawal } \\
\text { et al., (2017) }\end{array}$ & $\begin{array}{l}\text { Jeyakumari } \\
\text { et al., (2015) }\end{array}$ & $\begin{array}{l}\text { Rakesh kumar et } \\
\text { al., (2013) }\end{array}$ \\
\hline 33 & 33.3 & 40 & - \\
\hline 67 & 66. & 100 & - \\
\hline 100 & 100 & 100 & - \\
\hline 67 & 66.6 & - & 77 \\
\hline 33 & - & - & - \\
\hline 33 & - & - & - \\
\hline 67 & - & 100 & 95.5 \\
\hline 67 & - & - & 76 \\
\hline 100 & - & 100 & 90 \\
\hline 33 & - & - & - \\
\hline
\end{tabular}

Table.12 antibiotic susceptibility pattern of various organisms in CSOM

\begin{tabular}{|c|c|c|c|c|c|c|c|c|c|c|}
\hline Antibiotics & $\begin{array}{l}\text { Staph } \\
\text { Aureus }\end{array}$ & CoNS & $\begin{array}{l}\text { Citroba } \\
\text { cter }\end{array}$ & $\begin{array}{l}E \\
\text { coli }\end{array}$ & $\begin{array}{l}\text { Proteus } \\
\text { Vulgaris }\end{array}$ & $\begin{array}{l}\text { Proteus } \\
\text { mirabilis }\end{array}$ & $\begin{array}{l}\text { Klebsiella } \\
\text { pp }\end{array}$ & $\begin{array}{l}\text { Pseudomonas } \\
\text { ueruginosa }\end{array}$ & $\begin{array}{l}\text { Pseudo } \\
\text { mona } \\
\text { s spp. }\end{array}$ & $\begin{array}{l}\text { Non- } \\
\text { fermenters }\end{array}$ \\
\hline & $\%$ & $\%$ & $\%$ & $\%$ & $\%$ & $\%$ & $\%$ & $\%$ & $\%$ & $\%$ \\
\hline Erythromycin & 36 & 57 & - & - & - & - & - & - & - & - \\
\hline Clindamycin & 73 & 57 & - & - & - & - & - & - & - & - \\
\hline Cotrimoxazole & 55 & 14 & - & - & - & - & - & - & - & - \\
\hline Penicillin & 9 & 14 & - & - & - & - & - & - & - & - \\
\hline Tetracycline & 91 & 100 & - & - & - & - & - & - & - & - \\
\hline Linezolid & 100 & 100 & - & - & - & - & - & - & - & - \\
\hline Vancomycin & 100 & 100 & - & - & - & - & - & - & - & - \\
\hline Rifampicin & 100 & 100 & - & - & - & - & - & - & - & - \\
\hline Ciprofloxacin & 55 & 43 & - & - & - & - & - & - & - & - \\
\hline Gentamycin & 91 & 71 & 100 & 67 & 100 & 100 & 50 & 60 & 100 & 100 \\
\hline Ceftazidime & - & - & 0 & 33 & 50 & 100 & 50 & 100 & 100 & 100 \\
\hline $\begin{array}{l}\text { Ceftazidime/Clavulan } \\
\text { ic acid }\end{array}$ & - & - & 100 & 67 & 100 & 100 & 50 & - & - & - \\
\hline Meropenem & - & - & 100 & 100 & 100 & 100 & 100 & 80 & 82 & 100 \\
\hline Cefepime & - & - & 100 & 33 & 50 & 100 & 50 & 60 & 73 & 0 \\
\hline Ampicillin & - & - & 100 & 33 & 50 & 100 & 50 & - & - & - \\
\hline Amikacin & - & - & 100 & 67 & 100 & 100 & 100 & 60 & 64 & 100 \\
\hline Piperacillin & - & - & 100 & 67 & 100 & 100 & 50 & 80 & 91 & 100 \\
\hline $\begin{array}{l}\text { Piperacillin/Tazobact } \\
\text { um }\end{array}$ & - & - & 100 & 100 & 100 & 100 & 50 & 60 & 82 & 100 \\
\hline Cefazolin & - & - & 100 & 33 & 100 & 50 & 50 & - & - & - \\
\hline $\begin{array}{l}\text { Ticarcillin/Clavulanic } \\
\text { Acid }\end{array}$ & - & - & - & - & - & - & - & 100 & 91 & 100 \\
\hline Aztreonam & - & - & - & - & - & - & - & 80 & 91 & 100 \\
\hline Ciprofloxacin & - & - & - & - & - & - & - & 60 & 73 & 0 \\
\hline Colistin & - & - & - & - & - & - & - & 100 & 82 & 100 \\
\hline Polymyxin- B & - & - & - & - & - & - & - & 100 & 73 & 100 \\
\hline
\end{tabular}


Fig. No. 1 : Microorganism Isolated from CSOM

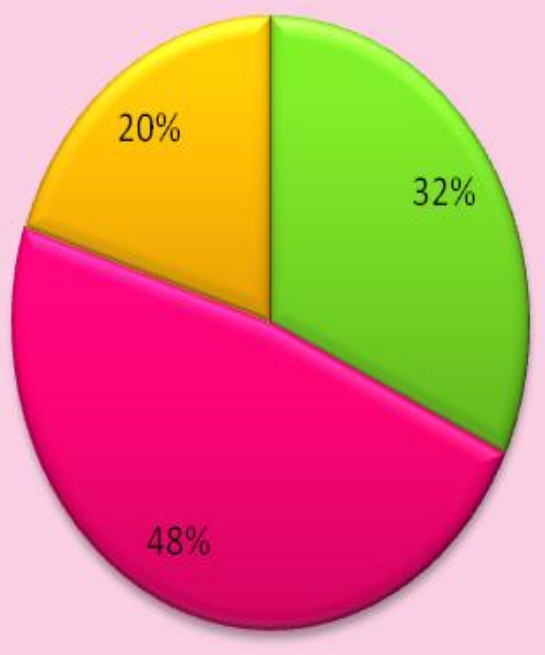

$\square \mathrm{GPC}$

$\square \mathrm{GNB}$

$\triangle F U N G U S$

Fig. No. 2 : Various species isolated from CSOM

Alterneria

1.79

Candida tropicalis

Aspergillus Niger

Candida Albicans

Nonfomenter

Klebsiella spp.

Proteus Vulgaris

Proteus Mirabilis

Pseudomonas aeruginosa

Citrobacter

E.coli

1.79

1.79

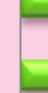

5.36

1.79

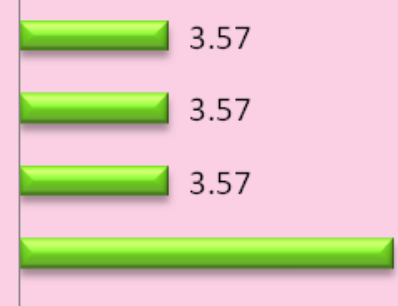

8.93

5.36

Pseudomonasspp.

CONS$$
1.79
$$

\section{Staphylococcus aureus}


Fig. No. 3 : Antibiotic Susceptibility Pattern of Various Organism Isolated in CSOM

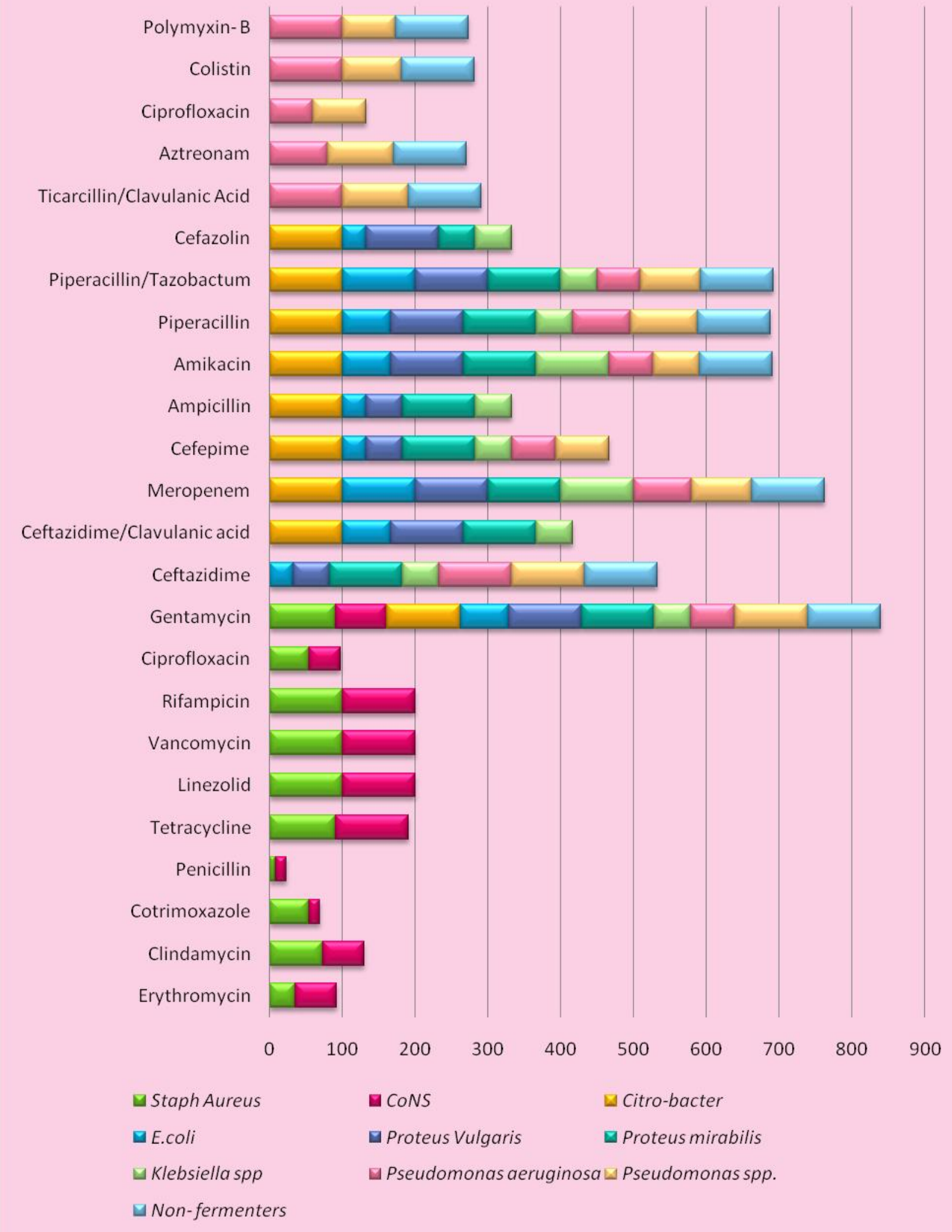




\section{Antibiotic sensitivity testing results}

Out of 11 Staphylococcus aureus isolates, all were sensitive to linezolid (100\%), vancomycin (100\%) and rifampicin (100\%). Staphylococcus aureus showed decreased sensitivity to gentamycin $(91 \%)$, tetracycline $(91 \%)$, clindamycin $(73 \%)$, cotrimoxazole $(55 \%)$, ciprofloxacin $(55 \%)$ and only erythromycin (36\%) and penicillin (9\%) sensitive.

Out of 7 CONS isolates all were sensitive to linezolid (100\%), vancomycin (100\%), rifampicin $(100 \%)$ and tetracycline $(100 \%)$. cons showed decreased sensitivity to gentamicin (71\%), erythromycin (57\%), clindamycin (57\%), ciprofloxacin (43\%) and only cotrimoxazole (14\%) and penicillin (14\%) sensitive.

In GNB, Citrobacter isolate was sensitive to all (100\%) except ceftazidime (0\%).

E. coli isolates, all were sensitive to meropenum $(100 \%)$ followed by ceftazidime/clavulanic acid (67\%), gentamycin (67\%), amikacin (67\%), piperacillin (67\%), ceftazidime (33\%), cefepime (33\%), ampicillin (33\%), cefazolin $(33 \%)$ (Table 11).

Proteus vulgaris isolates, all were sensitive to ceftazidime/clavulanic $\quad \operatorname{acid}(100 \%)$, meropenum $(100 \%)$, gentamycin $(100 \%)$, amikacin (100\%), piperacillin(100\%), piperacillin/tazobactum $(100 \%)$ and cefazolin $(100 \%)$ followed by ceftazidime (50\%), cefepime (50\%), ampicillin (50\%).

Proteus mirabilis isolates were sensitive to all $(100 \%)$ except cefazolin (50\%).

Klebsiella species isolates, all were sensitive to meropenum (100\%), amikacin (100\%) followed by ceftazidime ceftazidime/clavunic acid (50\%), gentamycin (50\%), cefepime $(50 \%)$, ampicillin $(50 \%)$, piperacillin $(50 \%)$, piperacillin/tazobactum (50\%), cefazolin $(50 \%)$.

In Pseudomonas aeruginosa isolates, all were sensitive to ticarcillin/clavulanic acid (100\%), ceftazidime $(100 \%)$, colistin $(100 \%)$ and polymyxin-b (100\%) followed by aztreonam $(80 \%)$, meropenem $(80 \%)$, piperacillin $(80 \%)$, cefepime (60\%), amikacin (60\%), ciprofloxacin $(60 \%)$, gentamycin $(60 \%)$ and piperacillin/tazobactum $(60 \%)$.

In Pseudomonas species isolates, all were sensitive to ceftazidime (100\%) and gentamycin (100\%) followed by ticarcillin/clavulanic acid (91\%), aztreonam $(91 \%)$, piperacillin (91\%), meropenem (82\%), piperacillin tazobactum (82\%), colistin (82\%), cefepime ciprofloxacin $(73 \%)$, polymyxin-B (73\%) and amikacin (64\%).

In the present study, age group of 11-20 years $(32 \%)$ were predominantly affected. This is similar to the study conducted by Rakesh Kumar et al., (2013) showed the increased prevalence of CSOM in 30-40 years age in his study. In our study second most prevalent age group was 21-30 years and 31-40 years 21\% each.

\section{Antibiotic sensitivity and resistance of CSOM}

In the present study, Out of 7 CONS isolates all were sensitive to linezolid (100\%), vancomycin $(100 \%)$ and rifampicin $(100 \%)$ and tetracycline (100\%). CONS showed decreased sensitivity to gentamicin $(71 \%)$, erythromycin (57\%), clindamycin (57\%), ciprofloxacin $(43 \%)$ and only cotrimoxazole (14\%) and penicillin (14\%) sensitive. which was correlated with the study of Ramesh Agrawal et al., (2017) showed 100\% sensitivity to linezolid, vancomycin (91\%), 
Clindamycin (91\%), Penicillin (27\%), Erythromycin (46\%) and Ciprofloxacin sensitive to $73 \%$ strains. A study conducted by Hirapure et al., (2014) showed $71 \%$ sensitivity to gentamicin and cotrimoxazole sensitive to 58\% cases (Table 12 and Fig. 3).

In the present study out of 2 Proteus mirabilis isolated, all were sensitive to all (100\%) except cefazolin (50\%). Proteus mirabilis was sensitive to gentamicin (95\%), chloramphenicol $(90 \%)$, tobramycin $(90 \%)$, cefotaxime (90\%), ceftriaxone (90\%), piperacillin tazobactum (85\%), and gatifloxacin (85\%). 100\% Proteus mirabilis isolated showed sensitivity to ceftazidime and ciprofloxacin. This was seen in study done by Mandana et al., (2011) and Osazuva et al., (2011) with similar sensitivity to Gentamicin.

\section{Acknowledgement}

The authors are thankful to the Department of Microbiology of National institute of medical sciences (NIMS) Medical College, Jaipur (Raj.) for providing necessary laboratory facilities to carry out this work.

\section{References}

Agrawal A, Kumar D, Goyal A, Goyal S, Singh N, Khandelwal G. 2013. Microbiological profile and their antimicrobial sensitivity pattern in patients of otitis media with ear discharge. Indian J. Otol., 19:5-8.

Asish J, Amar M, Vinay Hajare, Sreekantha, Avinash SS and Amareshar M. 2013 April. To study the bacteriological and mycological profile of chronic suppurative otitis media patients and their antibiotic sensitivity pattern. Int $J$ Pharm Bio Sci. 4(2): (b) 186 - 199.

Bansal Sulabh, Ojha Tarun, Kumar Suresh, Singhal Amit, Vyas Pratibha. 2013 Aug. Changing microbiological trends in cases of Chronic Suppurative Otitis Media. Patients Int J Cur Res Rev. Vol 05 (15) page 76.

Barobby GW, Zadik P. 1987. Bacteriology of otitis media in Ghana. Tropical Doctor. 17:91-92.

Berman S. 1995. Current concepts otitis media in children. $N$ Engl $\mathrm{J}$ Med. 332: 1560-5.

Bluestone CD, Klein JO. 1988. Otitis media in infants and children. Philadelphia: WB Saunders Co. 2.

Chakraborty, B., Subhadip, D., Debashish, G. 2014. Changing trends of antibiogram profile in patients with community acquired chronic otitis media in a tertiary care hospital. J. Evol. Med. Dent. Sci., 3(45): 11000-5.

Chandra J. 2008. Text book of medical mycology. $3^{\text {rd }}$ edn. New Delhi: Mehta Publishers. 266-290.

Chaudhary, B.L., Snehanshu Shukla. 2014. Bacteriological profile and their antibiotic susceptibility pattern in cases of otitis media. Bulletin of Pharmaceutical and Medical Sciences. Vol.2. Issue.2.

Clinical and laboratory standards institute (CLSI). 2017. Performance standards for antimicrobial Susceptibility testing, $27^{\text {th }}$ Ed Wayne, USA.

Dhingra, P.L. 2004. Dieases of ear, nose and throat; 3rd edition, chapter-4, Elsevier; $87-111$.

Dhirendra Kumar, Priyadarshini, M K Agarwal, $\quad$ P Prakash. 2016. Bacteriological Profile of Chronic Suppurative Otitis Media in Patients at a Tertiary Level Hospital. Eastern J Med Sci. Vol 1 (1).

Dr. Ramesh Agrawal, Dr. P.K. Khatri, Dr. R.S. Parihar, Dr. Harshada Shah. 2017. Microbial assessment of chronic Suppurative otitis media in a tertiary care centre of Rajasthan. international journal of health sciences and 
Research.Vol.7; Issue;2.

Geeta S. H. 2014 June. Study of Aerobes, Anaerobes and Fungi in CSOM in a Referral Hospital of Bangalore Rura. Journal of Evolution of Medical and Dental Sciences; Vol. 3, Issue 23, 09; Page: 6297-6303.

Hirapure P.V. and Pote M.K. 2014. Microbial Profile and Antibiograms of Active Patients of Chronic Suppurative Otitis Media in Latur, Maharashtra, India. State Reference Laboratory, Department of Microbiology, Government Medical College, Latur, MS, INDIA Vol. 2(5), 6-9,

Jeyakumari, D., Saranya, R., Nagajothi, J. and Selvalingam, E. 2015. Clinical and bacteriological profile of chronic suppurative otitis media in a rural area of Puducherry, India. International Journal of Development Research. Vol. 5, Issue, 09, pp. 5518-5

Jung TT, Hanson JB. 2006. Classification otitis media and surgical principles. Otolaryngeal Clin North Am; 39: 1221.

Jyothi Lakshmi, G., Geeta, Swarajya Lakshmi. 2014 December. Chronic Suppurative Otitis Media Profile of Aerobic Pathogens and Antibiotic Sensitivity. Journal of Evolution of Medical and Dental Sciences; Vol. 3, Issue 70,$15 ; 14957-14962$.

Koneman EW, Winn WC Jr. Allen SD, Janda WM, Procop GW, Schreckenberger PC, Woods GL. 2006. Koneman's colour atlas and text book of diagnostic Microbiology. $6^{\text {th }}$ edn. Philadelphia: Lippincot Williams and WIkins Company. Pp. 303-391.

Kumar H, Seth S. 2011. Bacterial and fungal study of 100 cases of Chronic Suppurative Otitis Media. J Clin Diagn Res. 5:1224-7.

Kusuma Bai S, Venkateswarlu K, Bala Krishna, Ashok reddy, Prasad Rao N. 2013. Study of bacteriology in chronic suppurative otitis media. Int $J$ Med Res

Health Sci. 2(3):510-513.

Madana J., Yolmo Deeke, Kalaiarasi.R. 2011. Microbiological profile with antibiotic sensitivity pattern of cholesteatomatous chronic suppurative otitis media among children. International Journal of Paediatric Otorhinolaryngol. 75(9): 1104-8.

Nawaz Umar, Bilal Ahmad Mir. 2015. Prevalence and Anti-Biogram of Pseudomonas aeruginosa Isolated from Otitis Media in KBN Hospital, Gulbarga. Sch. J. App. Med. Sci., 3(7A): 2502-2505.

Naz Ruby, Mohammad Khalid Farooqui, Girotra R. 2015. Bacterial profile and antibiotic sensitivity pattern of CSOM patient in Mewat region. $J$ Evid Based Med Health; 2(61), 9051-54.

Osazuwa F, Emmanuel Sazuwa, Clemnt Osines. 2011 Feb. Etiological agents of Otitis media in Benin city, Nigeria. North American Journal of Medical Science. Vol 3 (2).

Park DC, Lee SK. 2008. Antimicrobial resistance of Staphylococcus from Otorrhoea in chronic otitis media and comparison with results of all isolated Staphylococci. Eur J Clin Microbiol Infect Dis.527: 571

Parveen S, Rao R. 2012. Aerobic bacteriology of Chronic Suppurative Otitis Media (CSOM) in a teaching hospital. $J$ Microbiol Biotechnol Res.2: 586-590.

Prakash M. Lakshmi K, Anuradha S, Swathi G N. 2013. Bacteriological profile and their antibiotic susceptibility pattern of cases of Chronic Suppurative Otitis Media. Asian Journal of Pharmaceutical and Clinical Research. Vol 6, Suppl 3.

Prayaga N. Srinivas Moorthy, Jadi Lingaiah, Sudhakar Katari, Anil Nakirakanti. 2013. Clinical Application of a Microbiological Study on Chronic 
Suppurative Otitis Media International Journal of Otolaryngology and Head and Neck Surgery. 2, 290-294.

Raakhee T, Sreenivasa Rao Unguturu. 2014 May. Bacteriological study of discharging ear in patients attending a tertiary care hospital. International Journal of Research in Medical Sciences. 2(2):602-606.

Rakesh Kumar, P Srivastava, M Sharma, S Rishi, P S Nirwan, K Hemwani and S S Dahiya. 2013. Isolation and Antimicrobial sensitivity Profile of bacterial agents in Chronic Suppurative Otitis Media patients at NIMS hospital, Jaipur. International Journal of Pharmacy and Biological Sciences. 3(4): 265-269.

Roland PS, Meyerhoff WL. 1999. Opencavity tympanomastoidectomy. Otolaryngol Clin North Am; 32: 525

Shrestha B.L., Amatya R C, Shrestha I, Ghosh I. 2011. Microbiological profile of CSOM Vol 2.

Shyamala R, Reddy P, Sreenivasulu. 2012. The study of bacteriological agents of chronic Suppurative otitis mediaaerobic culture and evaluation. $J$. Microbial. Biotechnol. Res. 2(1): 15262.
Sunilkumar Biradar and C. Roopa. 2015. Study of Microbiological Profile and their Antibiogram in Patients with Chronic Suppurative Otitis Media Int.J.Curr.Microbiol.App.Sci. 4(9): 981985.

Susmita kumari sahu, Moningi venkata Narasimham, Indrani Mohanty, Sanghamitra padhi, Pritilata Panda, Banojini parida. 2014. Microbiological profile of chronic suppurative otitis media and in vitro antibiotic sensitivity pattern in a tertiary care hospital. Otolaryngology online journal. Vol. 4(4).

Upasana Bhumbla, Shobha Paul, Dinesh Raj Mathur and Gyaneshwari. 2016. Bacterial Study of CSOM and their Changing Patterns of Antibiotic Sensitivity and Resistance. Int.J.Curr.Microbiol.App.Sci. $\quad$ 5(10): 282-288.

Yadav Saurabh, Vaibhav. 2016. Study of Bacteriology of Chronic Suppurative Otitis Media and Its Recent Antibiotic Susceptibility Pattern. International Journal of Health Sciences and Research. Vol. 5(3).

\section{How to cite this article:}

Jitendra and Shiv Kumar. 2018. "Microbiological Profile and Antibiogram in Cases of Chronic Suppurative Otitis Media at a Tertiary Care Hospital, Jaipur." Int.J.Curr.Microbiol.App.Sci. 7(01): 395-407. doi: https://doi.org/10.20546/ijcmas.2018.701.045 